\title{
Stochastic Resonant Signaling in Enzyme Cascades
}

\author{
Yueheng Lan and Garegin A. Papoian* \\ Department of Chemistry, University of North Carolina at Chapel Hill, North Carolina 27599-3290, USA
}

(Received 18 January 2007; published 1 June 2007)

\begin{abstract}
We observe the phenomenon of stochastic resonant signaling in signal amplification enzyme cascades, where certain optimal reaction rates minimize the average threshold-crossing time. We develop a new analytical technique to obtain the mean first passage time, based on a novel decomposition of the master equation. Our analytical results are in good agreement with the exact numerical simulations. We demonstrate that resonant behavior may be a ubiquitous phenomenon in stochastic threshold crossing in cell signaling. The physical principles behind this phenomenon are elucidated.
\end{abstract}

DOI: 10.1103/PhysRevLett.98.228301

PACS numbers: 82.20.Uv, 82.40.Qt, 87.16.Xa

Introduction. - Signaling proteins often exist in a small number of copies in cells or subcellular compartments. Consequently, the discreteness in the number of reacting particles and the fundamentally random nature of chemical reactions lead to ubiquitous noise production. Thus, stochastic chemical dynamics is often invoked to describe cellular signaling processes, especially when noise qualitatively changes the system behavior [1-5]. For example, stochastic versus deterministic approaches predict different outcomes when modeling threshold crossing in specific biological reaction networks [6-11]. Interesting biological examples of threshold crossing induced by stochastic signals include the lambda phage infection [12] and the hierarchical enzymatic reactions in the mitogen-activated protein kinase cascades [13,14].

The main focus of this work is the problem of stochastic threshold crossing in nonlinear signal amplification cascades. We found that a noisy signal reaches the threshold fastest when the upstream and downstream reaction time scales are related in a specific way, indicating the existence of internal resonances embedded in cellular signaling cascades (see Fig. 1). This phenomenon is closely related to resonant activation which has been studied intensively and found interesting applications in physics, chemistry, and biology [15-19]. Our findings demonstrate how reaction rates of various nodes could be collectively tuned in protein signaling networks such that a signal is most efficiently picked up and transmitted through the network. We elucidate the physical nature of the observed resonant phenomenon with an approach based on the master equation and generating function description of stochastic chemical kinetics.

Because of the well-known difficulties in solving equations of stochastic kinetics, most prior analytical results on stochastic threshold crossing were obtained in the limit of either very short or very long correlation times [20,21]. In addition, noise was often modeled in the continuous limit, with the noise source considered of external origin $[15,16,22]$. To take into account the particle discreteness and the non-Gaussian character of the intrinsic noise, we develop here a novel analytical scheme which allows us to obtain approximately the mean first passage time. Our theory works well in the whole parameter regime, including the difficult cases where the upstream and downstream reaction time scales are not well separated.

The enzymatic signal amplification cascade, shown in Fig. 1(a), is one of the most fundamental elements in protein signal transduction networks, commonly found embedded in many important signaling cascades [14,23]. For instance, $R$ could represent an inactive kinase, which
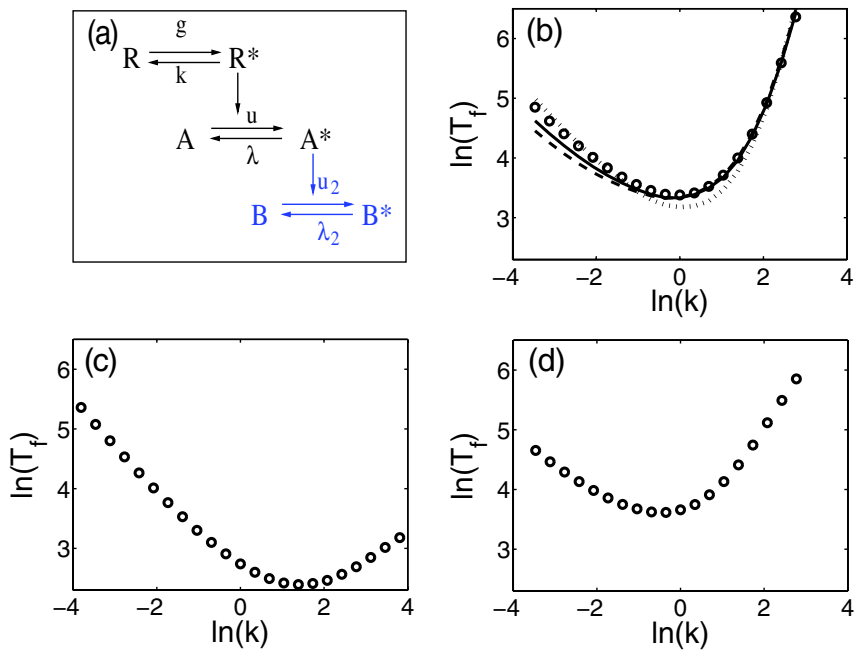

FIG. 1 (color online). (a) A 3-step cascade, where activation of $R^{*}$ in the upstream reaction, $R \rightarrow R^{*}$, results in subsequent activation of $A^{*}$ in the downstream reaction, $A+R^{*} \rightarrow A^{*}+$ $R^{*} . g=2 k, \mu=0.25, \lambda=1.5, \mu_{2}=0.1, \lambda_{2}=2.5$ with initial condition $\left(N_{R}, N_{R^{*}}, N_{A}, N_{A^{*}}, N_{B}, N_{B^{*}}\right)=(100,0,100,0,100,0)$; (b)-(d) Log-log plot of the first passage time $T_{f}$ vs the reaction rate $k$. (b) Comparison of different computations for the starting 2-step cascade in (a): Gillespie simulation $(\bigcirc)$, matrix diagonalization of Eq. (1) (dotted line), first order approximation Eq. (4) (dashed line), and second order approximation (solid line), with the $A^{*}$ critical value $n_{c}=50$. (c) Gillespie simulation as in (b) with the second reaction replaced by $R^{*}+A \rightleftharpoons A R^{*} \rightarrow$ $R^{*}+A^{*}$ and the critical value $n_{c}=35$. (d) For the full 3-step cascade with the $B^{*}$ critical value $n_{c}=75$. 
becomes activated into $R^{*}$ with a rate $g$. Alternatively, $R$ could be a surface receptor which is activated by binding of an external ligand. The activated kinase $R^{*}$ phosphorylates the next kinase $A$ downstream with a rate $\mu . A^{*}$ spontaneously decays to $A$ with a rate $\lambda$ and $R^{*}$ to $R$ with a rate $k$. The total number $N$ of $A$ and $A^{*}$ is a constant of motion. For simplicity, we assume that the $R$ activation is a Poisson process. Without this assumption, all the derivations below would still apply, with only minor modifications. We take the $A^{*}$ number average, $\bar{n}$, to be well below some predetermined critical value $n_{c}$. Rare fluctuations may bring $n$ over $n_{c}$, triggering further processes downstream. Henceforth, we study how the rare threshold crossings depend on the decay rate, $k$, when the average $R^{*}$ number, $\bar{m}=g / k$, is held fixed. Mathematically, we compute the first passage time $T_{f}$ for the number $n$ of protein $A^{*}$ to reach $n_{c}$ under the enzymatic influence from $R^{*}$. $T_{f}$ is a random variable which depends on $n_{c}$ and has a welldefined average, $\left\langle T_{f}\right\rangle$, the so-called mean first passage time (MFPT).

The probability distribution function (PDF) $P(m, n)$, of having $m R^{*}$ 's and $n A^{*}$ 's, and the related master equation may be used to describe its stochastic dynamics [24]. An absorbing boundary condition, $P\left(m, n_{c}\right) \equiv 0$, is used to model the threshold crossing. Therefore, there is a net probability flow out of the system and so the total probability $P_{t}=\sum_{m, n} P(m, n)$ decays exponentially in the longtime limit. The MFPT is calculated as $T_{f}=\int_{0}^{\infty} t\left(-d P_{t}\right)=$ $\int_{0}^{\infty} d t P_{t}$ (for notational simplicity we use $T_{f}$ instead of $\left\langle T_{f}\right\rangle$ here and later).

The master equation for $P(m, n)$ is defined on a twodimensional lattice in the $(m, n)$ plane. Direct computation of the corresponding eigenvalues requires diagonalization of a matrix with a dimension $(m+1)(n+1)$, which could be very expensive even for $m, n$ values of the order of hundreds. Below, we decompose the master equation into two equations, each defined on a one-dimensional lattice. This reduction of dimensionality plays a key role for the subsequent derivation of the analytical solution, also providing in-depth physical insights into the mechanism of the stochastic threshold-crossing process. An example of a long-time PDF, $P(m, n)$, is shown in Fig. 2(a). The absorbing boundary at $n=n_{c}$ induces a decay with rate $r_{m}$, accounting for the outgoing flow at $\left(m, n_{c}\right)$. Consequently, the total decay rate $\lambda_{1}$ is a function of these decay rates, $\left\{r_{m}\right\}$. If the decay is slow, the initial transient dynamics does not contribute significantly to the MFPT, which is instead determined by the asymptotic exponential decay. In the long-time limit, $P(m, n)$ is characterized by a stable profile, which diminishes exponentially with a constant rate, $P(m, n) \propto e^{-\lambda_{1} t}$.

Our new approach is to treat separately the $R-R^{*}$ and $A-A^{*}$ dynamics and, then, reincorporate back the interactions by using renormalized coupling variables. Thus, the derivation below contains three steps: (1) obtain the MFPT in terms of the rates $r_{m}$ [to be calculated in step (2)], (a)

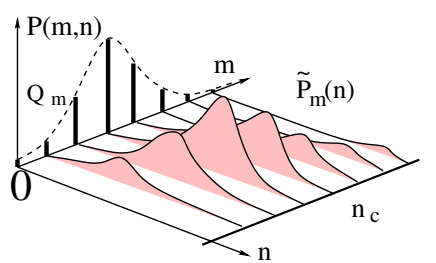

(b)

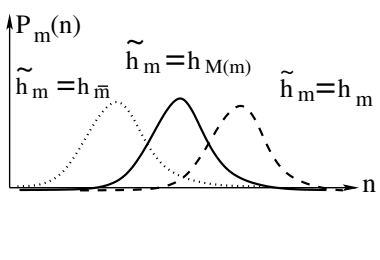

FIG. 2 (color online). (a) The PDF, $P(m, n)$, on the twodimensional lattice is shown. Filled curves indicate the conditional probability distribution $\tilde{P}_{m}(n)$ of $A^{*}$, for realizations with a specific number of activated $R^{*}$ receptors, $m$. The cutoff is shown at $n=n_{c}$ and the marginal probability $Q_{m}=\sum_{n} P(m, n)$ is shown with thick bars on the vertical panel; (b) the $A^{*}$ distribution for different $R^{*}$ switching rates: the large rate (dotted line for any $m$ ), the small rate (dashed line for $m>\bar{m}$ ), and the intermediate rate (solid line for $m>\bar{m}$ ).

(2) compute the decay rates $r_{m}$ with the $R^{*}$ number $m$ held stationary, (3) to take into account random switching among various $m$ states, modify each $r_{m}$ by introducing a renormalized variable $M(m)$.

First, we relate the MFPT to an eigenvalue equation in the $R-R^{*}$ dynamics. Let $Q_{m}=\sum_{n} P(m, n)$ denote the marginal probability for having $m R^{*}$ 's in the system [see Fig. 2(a)]. With absorption, each $Q_{m}$ satisfies an equation of the form:

$$
\frac{d Q_{m}}{d t}=g Q_{m-1}+k(m+1) Q_{m+1}-(g+k m) Q_{m}-r_{m} Q_{m},
$$

where the outgoing flux is modeled by the last term. In general, the leakage rate $r_{m}$ is a complicated, unknown function of time, which depends on both the $R^{*}$ and $A^{*}$ dynamics.

In the long-time limit, each $r_{m}$ relaxes to a specific timeindependent value. If all the $r_{m}$ 's were known, the eigenvalue problem of Eq. (1) could be solved numerically by direct matrix diagonalization, resulting in the dotted curve in Fig. 1(b). To obtain an analytical solution, we rely on successive approximations. Since the decay rates are slow, we use ansatz $Q_{m}=\exp (-g / k) \frac{(g / k)^{m}}{m !} f_{m}$ which gives the equilibrium $R^{*}$ distribution with no absorption when $f_{m}=$ 1 [24]. Substituting it into Eq. (1), an equation for $f_{m}$ is derived, which may be cast into an integral form

$$
\begin{aligned}
f_{m}= & e^{-\left(g+k m+r_{m}\right) t} \\
& \times\left[1+\int_{0}^{t} d t^{\prime} e^{\left(g+k m+r_{m}\right) t^{\prime}}\left(k m f_{m-1}+g f_{m+1}\right)\right],
\end{aligned}
$$

where $f_{m}(0)=1$ is used, corresponding to an initial equilibrium distribution for $R^{*}$. If the decay rate is small, then $f_{m}(t)=e^{-\lambda_{1} t}$ is a good zeroth order approximation, describing the asymptotic exponential decay with a fixed PDF profile. When substituted into Eq. (2), it leads to the first order solution, which then produces the MFPT $T_{f}=$ $1 / \lambda_{1}$ 


$$
1 / \lambda_{1}=\left\langle\frac{\left(r_{m}-\lambda_{1}\right) /\left(g+k m+r_{m}\right)+(g+k m) / \lambda_{1}}{g+k m+r_{m}-\lambda_{1}}\right\rangle .
$$

We can explicitly solve for $\lambda_{1}$

$$
\lambda_{1}=\left\langle\frac{r_{m}}{g+k m+r_{m}}\right\rangle /\left\langle\frac{1}{g+k m+r_{m}}\right\rangle .
$$

The notation $\left\langle l_{m}\right\rangle=\sum_{m=0}^{\infty} l_{m} \exp (-g / k)(g / k)^{m} / m$ ! denotes an average of any function of $m$. Second or higher order approximations may be obtained by using Eq. (2) recursively [solid line in Fig. 1(b)].

Our next task is to find the asymptotic values of $\left\{r_{m}\right\}$. If the number $m$ of $R^{*}$ is fixed and $P_{m}(n)$ denotes the conditional probability of having $n A^{*}$ 's, the generating function $\Phi_{m}=\sum_{n} P_{m}(n) y^{n}$ satisfies a first order linear partial differential equation

$$
\frac{\partial \Phi_{m}}{\partial t}=(1-y)\left(\lambda \frac{\partial}{\partial y}-\mu m N+\mu m y \frac{\partial}{\partial y}\right) \Phi_{m}-l_{m}(t) y^{n_{c}},
$$

where the last term was introduced to account for probability leakage at $n=n_{c}$, with $l_{m}(t)$ being an unknown rate which maintains $P_{m}\left(n_{c}\right)=0$. Starting with $n_{0} A^{*}$ 's, Eq. (5) without the last term has an approximate binomial solution

$$
\Phi_{m}(y) \approx\left[1+h_{m}(t)(y-1)\right]^{N},
$$

with

$$
h_{m}(t)=\frac{n_{0}}{N} e^{-(\lambda+\mu m) t}+\frac{\mu m}{\lambda+\mu m}\left(1-e^{-(\lambda+\mu m) t}\right) .
$$

$h_{m}$ is characterized by a relaxation time $\tau_{r}=1 /(\lambda+\mu m)$, beyond which $h_{m}$ approaches a steady value $\mu m /(\lambda+$ $\mu m)$, irrespective of the initial condition.

The rate $l_{m}(t)$ cancels the probability flow from the state $n=n_{c}-1$ to the boundary, and therefore can be written as $l_{m}(t)=\mu m\left(N-n_{c}+1\right) P_{m}\left(n_{c}-1\right)$. If the leakage is slow, in the long-time limit, $\Phi_{m}, P_{m}\left(n_{c}-1\right)$, and, thus, $l_{m}(t)$ decay exponentially. We may substitute the asymptotic forms $\Phi_{m}=\phi_{m} \exp \left(-r_{m} t\right), \quad l(t)=r_{m} \exp \left(-r_{m} t\right)$ into Eq. (5) and by canceling out the exponential time factor arrive at

$$
-r_{m} \phi_{m}=(1-y)\left(\lambda \frac{\partial}{\partial y}-\mu m N+\mu m y \frac{\partial}{\partial y}\right) \phi_{m}-r_{m} y^{n_{c}} .
$$

The constant factor $r_{m}$ before the exponential in $l(t)$ is chosen to satisfy Eq. (8) at $y=1$, since $\phi_{m}(1)=1$. The eigenvalue Eq. (8) can be solved explicitly

$$
\begin{aligned}
\phi_{m}(y)= & (\mu m+\lambda y)^{N-\left(r_{m} / \mu m+\lambda\right)}(1-y)^{r_{m} / \mu m+\lambda} \\
& \times\left[C+o\left(y^{n_{c}}\right)\right],
\end{aligned}
$$

where $C$ is a constant to be determined. Because of the absorbing boundary, the eigenfunction $\phi_{m}$ satisfies $P_{m}(n)=0$ for $n \geq n_{c}$. The term $o\left(y^{n_{c}}\right)$ in the square brackets on the right-hand side of Eq. (9) only contributes terms with $n>n_{c}$, so the condition $P_{m}\left(n_{c}\right)=0$ requires that the coefficient of $y^{n_{c}}$ outside the square brackets be equal to zero, which sets up the eigenvalue equation for $r_{m}$. We obtain $r_{m}$ by solving this equation numerically.

The final step is to consider the influence of the $R^{*}$ fluctuations on the leakage rate $r_{m}$. As investigated previously [24], the switching rate $k$ has a dramatic effect on the $A^{*}$ distribution, see Fig. 2(b). For the fast $R-R^{*}$ reaction, the relaxation time $\tau_{r}$ [defined after Eq. (7)] is large compared to the switching time $\tau_{s}$ (defined in the next paragraph) and effectively only the average $\bar{m}=g / k$ of $R^{*}$ is seen in the $A-A^{*}$ dynamics, so every reaction path gives an almost identical $A^{*}$ PDF [dotted curve in Fig. 2(b)]. Thus, in Eq. (7) $h_{m} \sim \bar{h}=\mu \bar{m} /(\lambda+\mu \bar{m})$ when $t \rightarrow \infty$, for all $m$ 's. In the case of slow $R-R^{*}$ reaction, $\tau_{r}$ is small compared to $\tau_{s}$ and the $A^{*}$ PDF closely follows each reaction path of $R^{*}$, such that $h_{m} \sim \mu m /(\lambda+\mu m)$ for each $m$ [dashed line in Fig. 2(b)]. In the intermediate regime, the $A^{*} \mathrm{PDF}$ is expected to lie between these two limits with some effective $\tilde{h}_{m}$ [solid line in Fig. 2(b)]. Based on the limiting functional form of $h_{m}$, we assume that a renormalized value $M(m)$ instead of $m$, determines $\tilde{h}_{m}$ through the defining equation

$$
\tilde{h}_{m}=\frac{\mu M(m)}{\lambda+\mu M(m)} .
$$

In a mean-field sense, we assumed that the asymptotic distribution $\tilde{P}_{m}(n)$ with absorption [see Fig. 2(a)] is also binomial and determined by $\tilde{h}_{m}$ through Eq. (6) and (7).

An analogy with quantum mechanics might be helpful. The $\bar{m}$ state corresponds to the "ground state" of the $R^{*}$ distribution. Other $m$ states (both below and above $\bar{m}$ ) are excitations with a finite (short) lifetime, reflecting smaller probability of their occurrence. An average excitation path starts from the ground state $(\bar{m})$, diffuses to the specified $m$ state in an average time $\tau_{m}$, remains there for $\tau_{\mathrm{ex}} \approx$ $1 /(g+k m)$, and then quickly decays back to $\bar{m}$ in a time $\tau_{d}$. The switching time $\tau_{s} \sim \tau_{m}+\tau_{\text {ex }}+\tau_{d}$. The "diffusion" time is approximated by $\tau_{m}=\alpha(m-g / k)^{2} /(g+$ $\mathrm{km}$ ), since the diffusion coefficient for the $R-R^{*}$ reaction is $g+\mathrm{km}$ [25]. $\alpha$ is a constant independent of $m, g$, and $k$ and is determined numerically by matching the numerical MFPT for a large $k$.

Most often $\tau_{m} \gg \tau_{\mathrm{ex}}, \tau_{m} \gg \tau_{d}$, so $\tau_{m} \approx \tau_{s}$ and it determines the value of $h_{m}$ in the excited states. Therefore, for the average path, $\tilde{h}_{m}$ is given by substituting $t=\tau_{m}$ and $n_{0}=N \bar{h}$ into Eq. (7),

$$
\tilde{h}_{m}=\bar{h} e^{-(\lambda+\mu m) \tau_{m}}+\frac{\mu m}{\lambda+\mu m}\left(1-e^{-(\lambda+\mu m) \tau_{m}}\right),
$$

from which the renormalized $M(m)$ is obtained using Eq. (10). Thus, in the intermediate regime, the $A-A^{*}$ reaction effectively "sees" instead of $m$ a renormalized value $M(m)$ and we should replace $m$ by $M(m)$ in Eq. (5), (8), and (9) when calculating $r_{m}$. As anticipated, $M(m)$ 
defined by Eq. (10) and (11) reproduces the limiting behaviors discussed above.

Using our approximation, we calculated the MFPT for different reaction rates $k$, keeping the average $\bar{m}=g / k$ fixed, and compared them with Gillespie computations over $10^{5}$ paths. As shown in Fig. 1(b), the MFPT curve from the analytical approximation agrees well with the numerical simulation results. The second order approximation (solid lines) improves perceivably over the first order (dashed lines). All the curves display a minimum, which signifies the existence of an optimal value of $k$, where the probability flow is most efficiently transmitted to the $n=n_{c}$ state. We also found that changing $\alpha$ mainly affects the MFPT curve corresponding to large $k$ values.

The previously mentioned three time scales play a major role in the threshold-crossing dynamics: (1) $\tau_{s}$, the $R-R^{*}$ switching time, (2) $\tau_{r}$, the relaxation time of the $A^{*}$ distribution, (3) $\tau_{c m} \sim 1 / r_{m}$, the characteristic leakage time. These time scales are intermingled and, in combination, determine the MFPT. $\tau_{s}$ controls how fast the receptor $R^{*}$ switches between different $m$ states. $\tau_{r}$ determines how quickly the PDF of $A^{*}$ follows the switching dynamics of $R^{*} . \tau_{c m}$ governs the frequency of a system visiting the absorbing boundary. Physically, when $k \rightarrow 0$, the $R-R^{*}$ switching time $\tau_{s}$ and, thus, MFPT go to infinity. When $k \rightarrow \infty$, the MFPT approaches a constant asymptotic value determined by $\bar{m}$, which is large in the current computation since the average $\langle n\rangle$ is assumed to be well below the cutoff $n_{c}$. The resonant signaling happens at some intermediate $k=k_{0}$, where the MFPT reaches a minimum. The optimal switching time for $R^{*}$ should be long enough for an average $A^{*}$ trajectory to diffuse to the boundary when $m>\bar{m}$, but short enough for $R^{*}$ to make frequent visits to different $m$ states, when $m<\bar{m}$. Thus, $k_{0}$ is located where the switching time of $R-R^{*}$ is about equal to the escape time through $n_{c}$ for a typical system $\left(k_{0} \sim 1\right.$ in Fig. 1). Consequently, when $\mu$ and $\lambda$ increase, the escape rate increases and, therefore, $k_{0}$ moves to larger values.

Analogous arguments have been used in the case of particles crossing a dichotomously fluctuating barrier $[15,18]$, but the physical picture of resonance in a nonlinear enzymatic cascade with discrete noise is significantly more complicated. Here, we have many discrete states and time scales. The noise is intrinsic and has very rich statistical features. Three key elements are necessary for the occurrence of the resonant signaling: stochastically switching enzymes, a downstream relaxation dynamics, and an absorbing boundary at the tail of the PDF. These conditions are often satisfied in many threshold problems of the biological networks. Therefore, we suggest that the stochastic signaling resonance is a ubiquitous phenomenon in cell signaling cascades. For example, the response curves also show similar nonmonotonic behavior for a 2-step cascade with Michaelis-Menten kinetics [Fig. 1(c)], a 3- step cascade without feedback [Fig. 1(d)], and with feedback (data not shown).

In summary, we discovered the phenomenon of stochastic resonant signaling in cellular enzyme cascades, where certain optimal reaction rates minimize the average signal propagation time. Our computations indicate that this phenomenon is ubiquitous, motivating systematic analytical and numerical investigations of the MFPT landscapes in various biologically relevant signal transduction cascades.

*Electronic address: gpapoian@unc.edu

[1] J. Paulsson, O. G. Berg, and M. Ehrenberg, Proc. Natl. Acad. Sci. U.S.A. 97, 7148 (2000).

[2] N. Barkai and S. Leibler, Nature (London) 403, 267 (2000).

[3] K. Wiesenfeld and F. Moss, Nature (London) 373, 33 (1995).

[4] M. B. Elowitz, A. J. Levine, E. D. Siggia, and P. S. Swain, Science 297, 1183 (2002).

[5] M. Thattai and A. van Oudenaarden, Genetics 167, 523 (2004).

[6] T. Lu, T. Shen, C. Zong, J. Hasty, and P. G. Wolynes, Proc. Natl. Acad. Sci. U.S.A. 103, 16752 (2006).

[7] G. M. Süel, J.G. Ojalvo, L. M. Liberman, and M. B. Elowitz, Nature (London) 440, 545 (2006).

[8] E. Aurell and K. Sneppen, Phys. Rev. Lett. 88, 048101 (2002).

[9] M. Freeman and J. B. Gurdon, Annu. Rev. Cell Dev. Biol. 18, 515 (2002).

[10] I. V. Maly, H. S. Wiley, and D. A. Lauffenburger, Biophys. J. 86, 10 (2004).

[11] T. Ushikubo, W. Inoue, M. Yoda, and M. Sasai, Chem. Phys. Lett. 430, 139 (2006).

[12] A. B. Oppenheim, O. Kobiler, J. Stavans, D. L. Court, and S. Adhya, Annu. Rev. Genet. 39, 409 (2005).

[13] C.-Y. F. Huang and J. J. E. Ferrell, Proc. Natl. Acad. Sci. U.S.A. 93, 10078 (1996).

[14] B. Schoeberl, C. Eichler-Jonsson, E. D. Gilles, and G. Müler, Nat. Biotechnol. 20, 370 (2002).

[15] C. R. Doering and J. C. Gadoua, Phys. Rev. Lett. 69, 2318 (1992).

[16] T. C. Elston and T. B. Kepler, Phys. Lett. A 280, 204 (2001).

[17] R. F. Fox and R. Roy, Phys. Rev. A 35, 1838 (1987).

[18] J. Maddox, Nature (London) 359, 771 (1992).

[19] R. D. Astumian and M. Bier, Phys. Rev. Lett. 72, 1766 (1994).

[20] J. M. Sancho, M. S. Miguel, S. L. Katz, and J. D. Gunton, Phys. Rev. A 26, 1589 (1982).

[21] P. Jung and P. Hänggi, Phys. Rev. Lett. 61, 11 (1988).

[22] J. Iwaniszewski, Phys. Rev. E 68, 027105 (2003).

[23] J.E. N. Pugh and T.D. Lamb, Biochim. Biophys. Acta 1141, 111 (1993).

[24] Y. Lan and G. A. Papoian, J. Chem. Phys. 125, 154901 (2006).

[25] D. T. Gillespie, J. Chem. Phys. 113, 297 (2000). 